\title{
Fuzzy Models for Fingerprint Description
}

\author{
Rosario Arjona ${ }^{1}$, Andrés Gersnoviez $^{2}$, Iluminada Baturone ${ }^{1}$, \\ ${ }^{1}$ Dept. Electrónica y Electromagnetismo (University of Seville), and Microelectronics \\ Institute of Seville (IMSE-CNM_CSIC), Isla de la Cartuja, 41092 Seville, Spain \\ \{arjona, lumi\}@imse-cnm.csic.es \\ ${ }^{2}$ Dept. de Arquitectura de Computadores, Electrónica y Tecnología Electrónica (University \\ of Cordoba), Cordoba, Spain \\ \{andresgm\}@uco.es
}

\begin{abstract}
Fuzzy models, traditionally used in the control field to model controllers or plants behavior, are used in this work to describe fingerprint images. The textures, in this case the directions of the fingerprint ridges, are described for the whole image by fuzzy if-then rules whose antecedents consider a part of the image and the consequent is the associated dominant texture. This low-level fuzzy model allows extracting higher-level information about the fingerprint, such as the existence of fuzzy singular points and their fuzzy position within the image. This is exploited in two applications: to provide comprehensive information for users of unattended automatic recognition systems and to extract linguistic patterns to classify fingerprints.
\end{abstract}

Keywords: Fuzzy modeling, automatic fingerprint identification systems, fingerprint classification, linguistic interpretability.

\section{Introduction}

A fingerprint image (Figure 1(a)) is a grayscale representation based on the reproduction of ridges (dark) and valleys (bright). Ridges and valleys are structural features originated by the exterior appearance of the epidermis [2]. Since fingerprints of a person are unique (even for twin brothers), they have been widely used for recognition purposes. The first stage of an automatic fingerprint recognition system is to acquire a digital image from the finger through a sensor. Then, the image is processed to extract several features. Those features are stored as the template of that fingerprint in the enrollment stage, or are compared (matched) with the template(s) in the recognition stage. The features extracted from the fingerprint can be global (if they provide information of the whole fingerprint) or local. An example of global features is the directional image (also called orientation image, field, or map, or directional field or map). It gives the local directions of ridges for each pixel or each block of pixels. Figure 1(b) shows the block directional image corresponding to the fingerprint in Figure 1(a). Examples of local features are minutiae. While local features are employed for final identification or authentication applications, global features are used as an aid at different stages such as enhancement and segmentation 


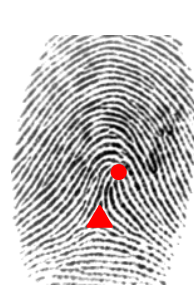

(a)

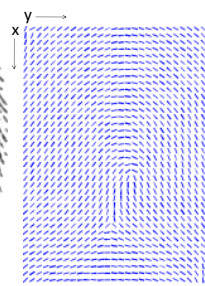

(b)

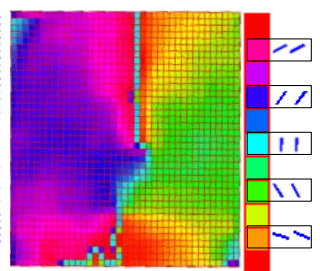

(c)

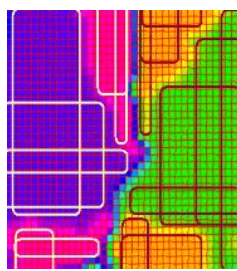

(e)

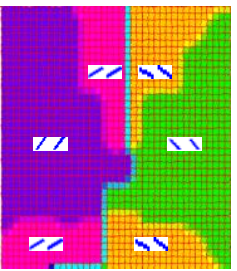

(f)

Fig. 1: (a) Fingerprint from FVC database [1] with singular points depicted (core as a circle and delta as a triangle). (b) Directional image associated. (c) Fuzzy model with 625 rules represented by xfplot. (d) Relations between directions and colors of the fuzzy model. (e)

Model with 4 clusters and the 16 most significant rules. (f) Model with 16 rules.

of fingerprint images, singular points extraction (core and delta, as shown in Figure 1(a)), alignment, matching, and fingerprint classification.

Fuzzy logic has been used for different purposes in fingerprint recognition systems such as fingerprint image enhancement [3], minutiae extraction and verification [4]-[5], matching [6]-[7], and classification [8]. The capability of fuzzy logic to cope with vagueness and uncertainty is typically exploited in these applications, mainly considering local features. This paper explores two other characteristics of fuzzy logic: capability of modeling and linguistic interpretability. These characteristics are employed to describe fingerprints as a whole. Hence, global features such as directional images are considered.

The paper is structured as follows. Section 2 introduces how to describe a fingerprint image by a fuzzy model. This process can be automated by using Matlab and the CAD tools of the design environment Xfuzzy 3 [9]. The model can be coarser or finer depending on the application. Section 3 illustrates the use of a coarse model to provide linguistically comprehensive feedback to the user of the fingerprint-based recognition system. Section 4 shows the applicability of fuzzy modeling to facilitate fingerprint classification. Finally, Section 5 gives some conclusions and summarizes future work.

\section{Fuzzy Modeling}

Since fuzzy systems are universal approximators, they have been used to model the behavior of systems from a set of representative numerical data that contain outputs associated to inputs. Fuzzy modeling has been typically applied to model controllers or plants to control, whose inputs and outputs take numerical values [10]. In this sense, the first step to model an image is to select which are the inputs and the outputs considered by the model. Recently, there is a wide research on applying fuzzy logic to images, because images contain imprecise and ambiguous information [11]. In particular, fuzzy models of images have been done considering low-level information, such as textures or grey or color values [12]. This is the approach taken herein: the directional image will provide the texture information about the image to be modeled. The fuzzy models pursued will have two inputs: the $\mathrm{x}$ and $\mathrm{y}$ coordinates 
of the pixel, and one output: the local direction of ridges at that pixel. The advantage of the fuzzy model will be its capability to approximate the pixel directional image with a low number of linguistically interpretable 'if-then' rules, that is, its capability of summarizing a large number of numerical data (directions of many pixels) into a small number of rules.

The design flow to obtain these fuzzy models has been automated by using several CAD tools. Firstly, an algorithm developed by [13] in Matlab creates gradient-based directional images from fingerprints. The pixel directional image has as many directions as pixels in the fingerprint image. For example, the fingerprint in Figure 1(a) has 374x276 pixels, which means a complete directional image with $374 \times 276$ directions (Figure 1(b) shows directional image sampled in blocks of 9x9 directions). All or a subset of these directions and their $x$ and $y$ positions are analyzed by the tool $x f d m$ of Xfuzzy 3 to extract fuzzy rules with two inputs ( $x$ and $y$ positions) and one output (direction between $0^{\circ}$ and $180^{\circ}$ ). This tool allows users to choose a grid- or clustering-based algorithm to extract fuzzy rules, the number and types of membership functions to cover inputs, and the conjunction and defuzzification operators used in the generated rule base. Since our objective are rule bases with linguistic interpretability, the grid-based Wang-Mendel algorithm has been selected with product as conjunction operator, and Max Label for defuzzification (which selects the consequent of the most activated rule as the output of the system). Initially, the model is finer, for example using 25 Gaussian membership functions for each input. In this case, the generated system is composed by 625 rules $(25 \times 25$ combinations of antecedents), each one with its corresponding consequent. Since the membership functions cover the inputs uniformly, a group of $15 \times 11$ pixels is approximately described by a rule, which is something similar to compact the pixel directional image (with $374 \times 276$ directions) by the block directional image (with 625 blocks of $15 \times 11$ directions). The resulting model is shown by the tool xfplot of Xfuzzy 3 which displays the output of the system (direction) depending on $x$ and $y$ positions (Figure 1(c)). Meaning of colors in fuzzy model regarding directional image can be viewed in Figure 1(d), where directions selected (from bottom to up) correspond to the following fuzzy sets: almost horizontal in counter-clockwise, almost vertical in clockwise, vertical, almost vertical in counter-clockwise, and almost horizontal in clockwise.

Although the model with $25 \times 25$ rules reduces information by more than two orders of magnitude, the number of rules can be further reduced if non relevant information is eliminated. This can be done if the values of the directions (the 625 consequents of the rules) are described by a given set of representative directions (let us say 3,4 , etc., depending on the coarseness desired for the model: finer models as more prototypes selected). A way to find prototypes among a set of values is to apply clustering algorithms on those values. The tool $x f s p$ of Xfuzzy 3 allows applying Hard $C$-means clustering on the consequents, with the number of clusters chosen by the user. Fuzzy models that use such clustering produce a segmented directional image, for example, the 4 clusters shown in Figure 1(f) correspond to those depicted in Figure 1(d) except for the vertical. The rules whose consequents are the same and antecedents are represented by neighbor membership functions can be merged into a rule covering more situations. This merging can be also automated by the tool $x f_{s} p$ 
[14]. For example, when consequents are clustered into 4 groups, the 625 rules can be merged into 61 rules.

If the model is intended to be linguistically interpretable by a human, the number of rules should be small. A way to achieve this objective is to take the most significant rules for each consequent prototype (or exclude the least significant ones) as proposed in [12]. The idea is to select those rules obtained from merging more atomic rules. Such simplification allows reducing also the number of fuzzy sets employed to describe the $x$ and $y$ positions. For example, considering the model with 61 rules commented above, a new model with 16 rules could be achieved (Figure $1(\mathrm{f})$ ). The resulting model provides a linguistic description simple and intuitive because the fuzzy sets considered for antecedents and consequents are only a few and have clear linguistic meaning. Examples of the rules in this model are the following:

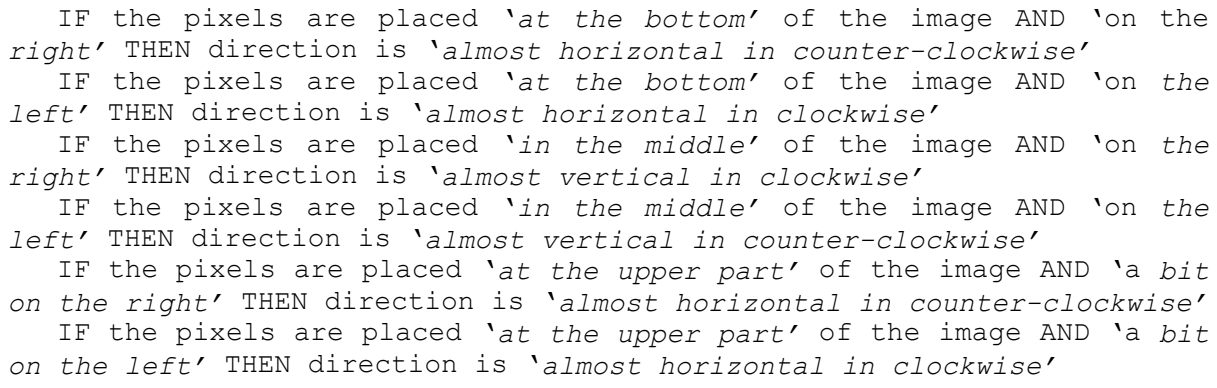

The model with 16 rules contains an incomplete rule base, because there are combinations of input fuzzy sets that are not considered by any rule. Anyway, the pixels placed at those positions will have the directions assigned by the closest defined rule with higher activation degree, as a result of using fuzzy sets that describe the pixel positions. This is illustrated in Figure 1(f), where all the pixels have an associated direction. Concerning approximation, the different models provide the following Root Mean Square Errors (RMSE):

- $\quad$ Model with 625 rules, RMSE $=0,060$

- $\quad$ Model with 61 rules, RMSE $=0,067$

- $\quad$ Model with 16 rules, RMSE $=0,112$

\section{Fuzzy Feedback to Users of Recognition Systems}

Many fails of automatic fingerprint-based recognition systems are due to a poor acquisition of user fingerprints. For example, the user may apply too few or too high pressure with the finger on the sensor, or the finger may not be correctly placed for the sensor to capture a good image. This issue is already considered by many sensors that inform the user with a red led if acquisition goes bad or a green led if everything goes well. Such feedback is too crisp and does not guide the user to improve acquisition. Fuzzy feedback with more linguistically interpretable information would be very useful for fully automatic unattended fingerprint-based recognition systems. If fingerprint acquisition is improved, complexity of the automatic recognition system 


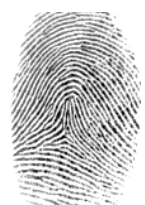

(a)

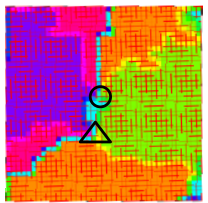

(g)

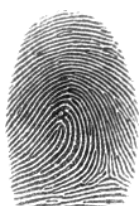

(b)

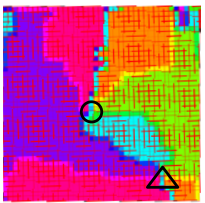

(h)

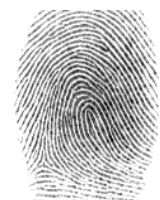

(c)

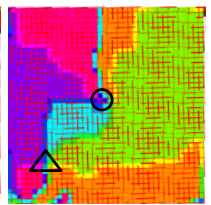

(i)

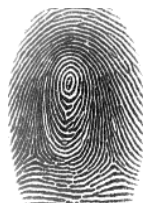

(d)

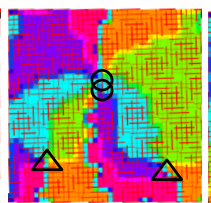

(j)

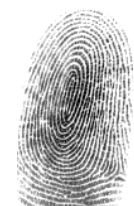

(e)

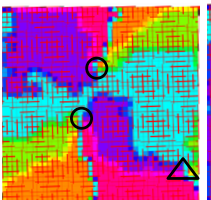

(k)

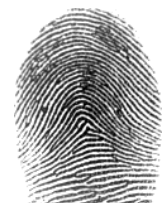

(f)

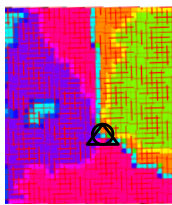

(1)

Fig. 2: (a) tended arch, (b) left loop, (c) right loop, (d) whorl, (e) whorl and (f) arch. (g) to (l) Models based on 5 clusters for the fingerprints in (a) to (e) with singular areas detected: (g)-(i) one convex core and one delta, (j) one convex-concave core and two deltas, (k) one convex and concave core and one delta, and (1) one convex-delta area.

can be reduced drastically. For example, fingerprint classification can be done with a simple fuzzy inference system, as described in the following section.

Our proposal for fuzzy feedback is based on detection or not of singular points (cores and deltas) and their location within the fingerprint acquired. Depending on this, users have to move horizontally or vertically their fingers. The fuzzy models extracted as commented above have been employed for this purpose. The number of clusters for the rule's consequents and the partition of antecedents is fixed by using models which allow detecting singular points in different groups of fingerprints. Let us consider the fingerprint classification proposed by Galton in [15] and extended by Henry in [16] that discriminates five groups: tended arch, left loop, right loop, whorl and arch. Figures 2(a)-(f) displays examples of fingerprints from FVC database [1] and 2(g)-(1) are fuzzy models with 5 clusters and a $25 \times 25$ partition (this selection offers a good trade-off between coarseness of the models and simplicity of the results). Singular areas of these models are where direction regions intersect. Since fuzzy models are coarse, such areas contain or are close to the singular points traditionally used in fingerprint recognition: convex core, concave core and delta. In order to detect these areas, five fuzzy patterns are considered as illustrated in Figure 3(a)-(e): convex core, concave core, delta, convex-concave and convex-delta. They are composed by the five clusters of directions combined with certain order. The corresponding areas in the models can be viewed in Figures 2(g)-(l). The singular areas convex-concave (in Figure 2(j)) and convex-delta (in Figure 2(1)) are the result of fuzzy merging a convex and a concave core, and a convex core and a delta, respectively. Detection of these singular areas is carried out by analyzing the direction values (consequents) of neighbor rules of the model. Fingerprints can be acquired with a certain inclination, so the detection of singular areas considers possible rotations (to a certain extent so as to distinguish, for example, between convex and concave cores). 


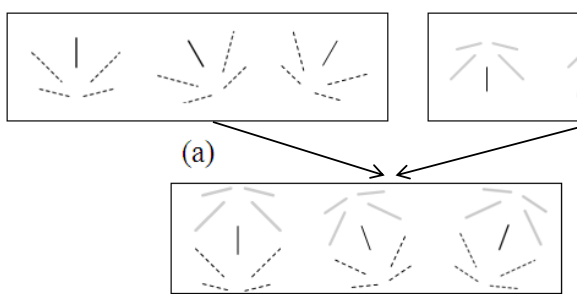

(d)

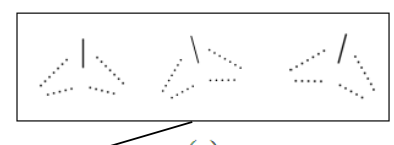

(c)

Fig. 3: Fuzzy patterns for detection of singular areas: (a) concave core. (b) convex core. (c) delta. (d) convex-concave core. (e) convex-delta.

Location of these singular areas within the image gives information to know how acquired fingerprint is. Let us consider, for example, the fingerprints depicted in Figures 4(a)-(f) and their fuzzy models in Figures 4(g)-(l). These fingerprints are not well captured because singular areas are shifted or they do not appear. Fuzzy feedback consists in communicating to the user what type of movement has to be applied to introduce the finger in a correct way. The detection of singular areas is followed by a fuzzy inference engine based on rules that generates a message for the user. This fuzzy inference engine employs the following fuzzy sets for the fuzzy patterns positions: very much on the left and very much on the right for the horizontal position of the image (Figure 5(a)), and very much at the bottom and very much at the top are considered in the vertical position (Figure 5(b)). The rules included in this fuzzy inference engine are that 'if any of the singular areas is placed in one or two of these fuzzy positions, the user is required to move the finger in the opposite direction'. For example, in Figure 4(g) the convex-delta is placed very much on the left, hence the system requires that the user moves the finger on the right. In Figure 4(e) there is not singular area, therefore the fuzzy inference engine requires the user puts the finger on the sensor again. In any other case, the engine decides that the finger is well placed.

\section{Fuzzy Models for Fingerprint Classification}

Fuzzy models can be also exploited for classification purposes. Automated fingerprint identification systems have to match a capture with all the fingerprints stored in a database. A large number of comparisons are necessary. To accelerate this process, fingerprint classification gives information to split databases and search only in the selected group of fingerprints. Classification of fingerprints has been done using several techniques such as crisp (non-fuzzy) rule-based, syntactic, structural, statistical, neural network, and combined techniques [2]. The technique proposed herein is to employ fuzzy inference using singular areas (as commented in the previous sections) to distinguish groups of fingerprints by applying again a subsequent fuzzy inference engine. Each group is characterized by the types, locations and number of singular areas. For example, in Figure 2(a)-(c), tended arch, left loop and right loop have one convex core and one delta. However, they are differenced by 


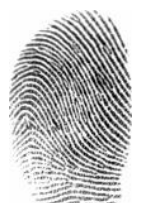

(a) (b)

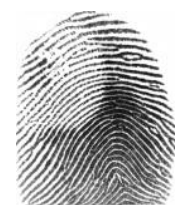

(c)

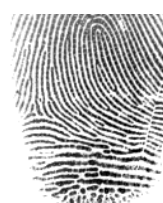

(d)

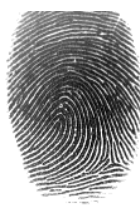

(e)

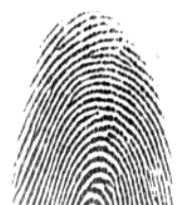

(f)

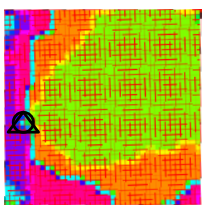

(g)

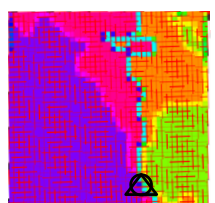

(h)

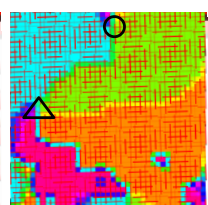

(i)

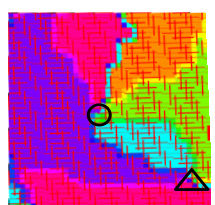

(j)

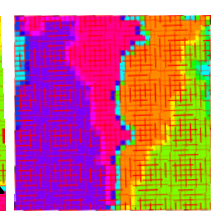

(k)
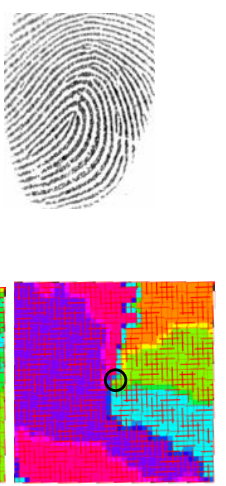

(1)

Fig. 4: Fingerprints captured with faults: (a) convex-delta area shifted very on the left. (b) convexdelta area shifted very at the bottom. (c) convex core shifted very at the top. (d) delta shifted very on the right. (e) there is not singular area. (f) there is not delta area. (g)-(l) Fuzzy models associated with 5 clusters.

delta position with respect to convex core: aligned, on the right and on the left, respectively. Although whorl group has two deltas, one convex core and one concave core (in Figure 2(d)); or one delta and one convex-concave core (in Figure 2(e)), the detection of such cores is enough to classify a fingerprint as a whorl. Arch case needs to detect the convex-delta area. Rule base for this classification system is shown in Table 1. Figure 5 (c) illustrates the fuzzy sets for the inference of the position of delta with respect to convex core in tended arch, left or right loop fingerprints. If the fingerprint has been acquired correctly (following the feedback commented in the previous section) the percentage of right classification can be $100 \%$. This has been proven with fingerprints from FVC 2002 [1].

Table 1. Rule base for the classification system.

\begin{tabular}{|c|c|c|c|}
\hline IF & singular areas & $\begin{array}{c}\text { position of delta with } \\
\text { respect to convex core }\end{array}$ & THEN \\
\hline 1$)$ & (core-delta $)$ & - & arch \\
\hline 2$)$ & (convex AND concave) OR (convex-concave) & - & whorl \\
\hline 3$)$ & (convex AND delta) & aligned & tended arch \\
\hline 4$)$ & (convex AND delta) & right & left loop \\
\hline 5$)$ & (convex AND delta) & left & right loop \\
\hline
\end{tabular}

\section{Conclusions}

Coarse fuzzy models of fingerprints can be analyzed by subsequent fuzzy inference engines to provide useful feedback information for users of automatic recognition systems and to understand classification results. A future work is to analyze if finer models could be exploited for identification or authentication purposes. 


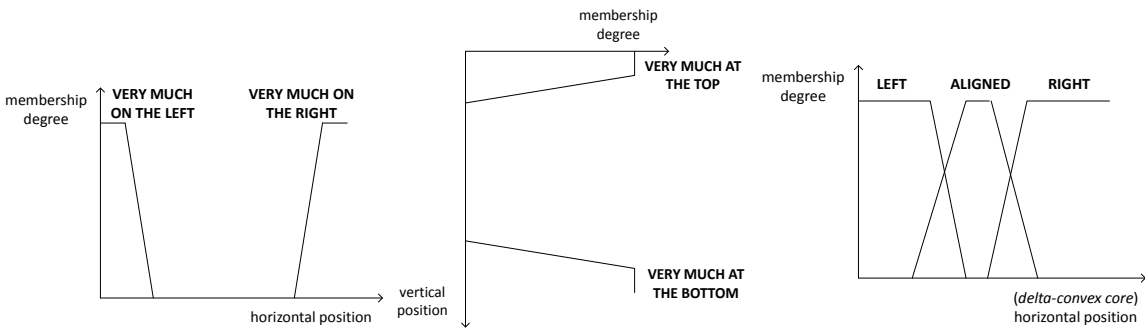

Fig. 5: Fuzzy sets for: (a) horizontal position and (b) vertical position of singular area in image. (c) position of delta area with respect to convex core.

\section{References}

1. FVC database, http://bias.csr.unibo.it/fvc2002/

2. Maltoni, D., Maio, D., Jain, A. K., Prabhakar, S.: Handbook of Fingerprint Recognition. 2nd ed., Springer (2009).

3. Bansal, R., Arora, P., Gaur, M., Sehgal, P., Bedi, P.: Fingerprint image enhancement using type-2 fuzzy sets. Proc. Sixth Int. Conf. on Fuzzy Systems and Knowledge Discovery, pp. 412-417. Tianji (2009).

4. Sagar, V.K., Alex, K.J.B.: Hybrid Fuzzy Logic and Neural Network Model for Fingerprint Minutiae Extraction. International Joint Conference on Neural Networks, pp. 3255-3259. Washington (1999).

5. Vert, G., Nasser, S.: Minutia Verification using Fuzzy Logic and Geographic Points of Reference. The 14th IEEE Int. Conf. on Fuzzy Systems, pp. 839-844. Reno (2005).

6. Yuan, L.: Application of Fuzzy Pattern Recognition on Fingerprint Processing. Second International Conference on Intelligent Computation Technology and Automation, pp. 660-663. Changsha (2009).

7. Chen, X., Tian, J., Yang, X.: A New Algorithm for Distorted Fingerprints Matching Based on Normalized Fuzzy Similarity Measure. IEEE Trans. on Image Processing, 767776 (2006).

8. Conti, V., Perconti, D., Romano, S., Tona, G., Vitabile, S., Ganglio, S., Sorbello, F.: Fast Fingerprint Classification Only Using the Directional Image. Lecture Notes in Computer Science, Volume 4692, pp. 34-41. Knowledge-Based Intelligent Information and Engineering Systems (2007).

9. Xfuzzy Web page, http://www.imse-cnm.csic.es/Xfuzzy/

10. Kosko, B.: Fuzzy Systems as Universal Approximators. IEEE Transactions on Computers, Vol. 43, No. 11, pp. 1329-1333 (1994).

11. Nachtegael, M., Van der Weken, D., Kerre, E.E.: Soft Computing Image Processing: Recent Advances. Series Studies in Fuzziness and Soft Computing. Springer-Verlag, (2007).

12. Baturone, I., Gersnoviez, A.: Automatic Extraction of Linguistic Models for Image Description. IEEE International Conference on Fuzzy Systems. Barcelona (2010).

13. Code for directional image, http://www.csse.uwa.edu.au/ pk/Research/MatlabFns/index.html

14. Baturone, I., Moreno-Velo, F. J., Gersnoviez, A.: A CAD Approach to Simplify Fuzzy System Descriptions. International Conference on Fuzzy Systems, pp. 2392-2399. Vancouver (2006).

15. Galton, F.: Finger Prints. Macmillan and co. and New York (1892).

16. Henry, E.: Classification and Uses of Finger Prints. Routledge (1900). 